Die in der letzten Zeit durchgeführten anatomisch-entwicklungsgeschichtlichen Untersuchungen an mehreren $B e y-$ tolomia- und Sonerila-Arten (Melastomataceen) konnten zeigen, $\mathrm{daB}$ in frühen Entwicklungsstadien, vor der Anlage der Silberflecken, das Anthocyan gleichmäßig oder nahezu gleichmäßig über die Lamina verteilt ist. Die zu den Silberflecken führende Interzellularbildung findet danach, und zwar immer vor dem ersten Auftreten von Differenzen im Anthocyangehalt statt, was mit unserer vorhin genannten Hypothese vollkommen übereinstimmt. Allerdings stellen diese Ergebnisse noch keinen Beweis für die Schattenwirkung dar, da Unterschiede im Anthocyangehalt auch in noch völlig gefalteten Blättern vorkommen, in denen die Interzellularen der Oberseite auf keinen Fall einen Schatten werfen können.

Auf experimentellem Wege konnte dagegen ein Beweis exbracht werden. Die Versuche sind mit Begonia lubbevsii ${ }^{1}$ ) durchgeführt worden. Zunächst wurde versucht, die Blätter nur von unten $z \mathrm{u}$ beleuchten, was aber mißlang, weil sie sich immer mit der Blattoberseite dem Licht zudrehen. Hinderte man sie an dieser Drehung, so starben die Blätter innerhalb

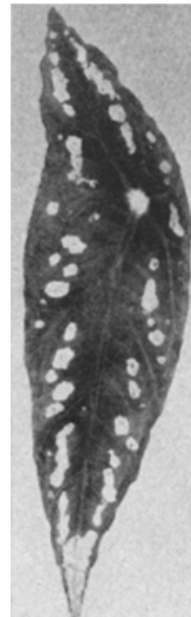

Fig. 1 a

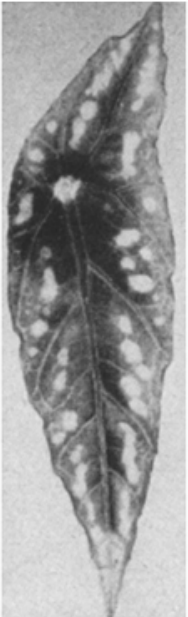

Fig. 1 b

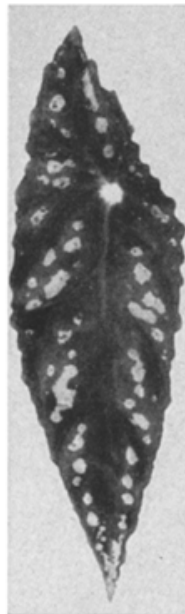

Fig. $2 \mathrm{a}$

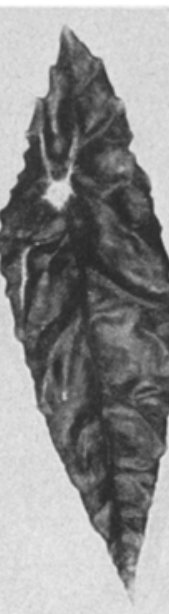

Fig. $2 \mathrm{~b}$
Fig. 1 a u. b. a Oberseite eines nur von oben belichteten Blattes von Begonia lubbersii mit Silberflecken; b Unterseite desselben Blattes, die Bereiche unter den Silberflecken (hell) enthalten kein Anthocyan

Fig. 2 a w. b. a Oberseite eines allseitig belichteten Blattes mit Silberflecken; b Unterseite desselben Blattes, gleichmäBig rot gefärbt

weniger Tage ab. Die Pflanzen wurden darum in einem Lichtkasten allseitig gleichmäßig beleuchtet. Bereits bei der Entfaltung zeigten die jungen Blätter solcher Pflanzen zunächst einen etwas schwächeren Anthocyangehalt unter den Silberflecken als im übrigen Bereich, wie dies auch unter normalen Bedingungen der Fall ist. Bei Kontrollpflanzen, die im gleichen Lichtkasten aufwuchsen, bei denen das Licht aber von der Seite und unten abgeschirmt war, verstärkte sich diese Differenz immer mehr, bis die zu den Silberflecken korrespondierenden Areale der Blattunterseite völlig grün erschienen (Fig. 1a, b). Die allseitig beleuchteten Pflanzen hingegen zeigten unter den Silberbereichen eine kontinuierliche Zunahme des Anthocyangehalts, so daß schließlich die Unterseite gleichmäßig rot gefärbt war (Fig. 2a, b). Eine Veränderung des Flavonolgehalts (Rutin) in diesen Blattabschnitten trat dabei nicht auf. Während also bei ausschließlicher Beleuchtung von oben das Anthocyan unter den Silberflecken abgebaut wird und diesem Abbau infolge zu geringer Lichtintensität keine Neusynthese gegenübersteht, findet eine Anthocyanneubildung in ausreichender Menge statt, sofern die entsprechenden Zellen direktes Licht erhalten. Diese Beeinflussung des Anthocyangehalts ist aber nur während der Entwickiung der Blätter möglich, denn ältere Blätter, die zur Zeit der Úberführung in den Lichtzasten schon voll entfaltet waren, zeigen keine Veränderung ihrer Zeichnung mehr.

Das Fehlen des Farbstoffes unter den Silberflecken bei erwachsenen Blättern ist danach in erster Linie auf die relativ geringe Lichtintensität, die in den unteren Schichten dieser Blattbereiche herrscht, zurückzuführen. Darauf weist bereits die früher mitgeteilte Tatsache hin, daß die Unterschiede im Winter bei schwacher Beleuchtung deutlicher ausgeprägt sind als bei stärkerer Beleuchtung im Sommer. Es gibt zwar einige Formen und Familien (Marantaceen, Commelinaceen), bei denen nie eine Beeinflussung der Anthocyanverteilung durch Silberzeichnung auftritt. Das spricht aber nicht gegen unsere Annahme, denn eine Reihe von Pflanzen bzw. Pflanzenorganen vermag auch im abgeschwächten Licht oder sogar im Dunkeln Anthocyan zu synthetisieren (z.B. die Rhizome mancher Araceen), wozu die meisten anderen Pflanzen nicht in der Lage sind.

Die genannten Ergebnisse können als eindeutig angesehen werden, reichen aber nicht für eine völlige Erklärung der Erscheinung aus. Es besteht nämlich im Verlauf der Anfangsentwicklung der Silberflecken offenbar ein zusätzlicher $\mathrm{Zu}$ sammenhang zwischen Interzellularenbildung und der Anthocyansynthese in den Zellen der entsprechenden Bereiche, der vielleicht mit der hier herrschenden Stoffwechselveränderung zusammenhängt.

Die Untersuchungen wurden durch eine Sachbeihilfe der Deutschen Forschungsgemeinschaft unterstützt.

Botanisches Institut der Universität, Freiburg i.Br. Martin Bopp und Gerhard Tauchmann Eingegangen am 26. Februar 1958

1) Bopr, M.: Planta 48, 631 (1957). - Ber. dtsch. bot. Ges. 69 , (18) (1956). $-{ }^{2}$ ) STAHL, E.: Ann. Jard. bot. Buitenzorg 13, 137 (1896). - 3) WIEBALK, G.: Z. Bot. 45, 175 (1957). - BERGDor.T, B.: Z. Bot. 43, 309 (1955). - 4) BARTH, H.: Planta 49, 103 (1957) (vgl. S. 121).

\section{Notiz zur Wirkung eines Cortisonderivates}

$4^{1}$-3, 11, 20-Triketo-17, 21-dioxy-pregnen-21-acetat (I) ist als ein Cortison-(II)-Derivat aufzufassen, was durch die Möglichkeit der Benennung als 1-Dehydro- $4,5 \beta$-dihydro-cortison21 -acetat verdeutlicht werden kann. (I) wurde von KE.NDALL und Matrox ${ }^{1}$ ) beschrieben, ohne daß über die biologische Aktivität Befunde bekannt wurden. Die Wirsamkeit von (I) und (II) - beide als Acetate - zur Thymusinvolution an adrenalektomierten Mäusen verhält sich wie $2: 1$ (ED $50_{\mathrm{I}}=$ $0,89 \mathrm{mg} ; E D 50_{\mathrm{II}}=0,45 \mathrm{mg}$ ). Ebenfalls wie $2: 1$ ist die Aktivität von (I) und (II) hinsichtlich der Glykogenspeicherung in der Leber bei adrenalektomierten Ratten. Die gleiche Verbältniszahl ist über die Schułzwirkung gegen die Vergiftung mit Hühnereiweiß (intraperitoneale Injektion an Ratten) zu errechnen. (I) ist im Uberlebenstest an adrenalektomierten Ratten $(0,2 \mathrm{mg} /$ täglich) voll wirksam. - Die ausführlichen Befunde werden wir (gemeinsam mit J. GebHard, K. Helrer und G. KLoTz) in den Acta Endocrinologica veröffentlichen. G. LANGBEIN verdanken wir die Herstellung der Substanz.

Laboratorien des VEB Jenapharm, Jena $i$. Thïrngen

Eingegangen am 27. März 1958

F. HOHENSEE

1) Kendali, E.C., u. V.R. Mattox: J. Biol. Chem. 188, 287 (1951).

Der Einfluß von Thyroxin und 2,4-Dinitrophenol auf die $\mathbf{N}_{2} \mathbf{O}$-Narkoseschwelle schilddrüsenloser Ratten

Thyroxin erhöht die Narkoseschwelle bei Meerschweinchen um $\left.62 \%^{1}\right)$. Eine entsprechende Senkung der Schwelle durch Methylthiourazil gelang nicht. Um eine sichere Auskunft auf die Frage zu erhalten, ob bei Thyroxinmangel die Narkoseschwelle nicht doch konsequent absinkt, wurde bei Ratten vor und nach operativer Entfernung der Schilddrüse die $\mathrm{N}_{2} \mathrm{O}$ Narkoseschwelle manometrisch bestimmt [Methode s. ${ }^{2}$ ]]

Der in der Überdruckkammer gemessene $\mathrm{N}_{2} \mathrm{O}$-Partialdruck, bei dem Stell- und Haltereflexe des Versuchstieres so weit ausgefallen waren, daß es sich aus Seiten- oder Rückenlage bei dreimaliger Prüfung nicht innerhalb von $20 \mathrm{sec}$ wieder aufzurichten versuchte, wurde als $\mathrm{Ma} \beta$ für die Narkoseschwelle angesehen. Als Versuchstiere dienten 44 weiße, männliche Ratten im Gewicht von 180 bis $260 \mathrm{~g}$, und insgesamt wurden 137 Einzelbestimmungen der $\mathrm{N}_{2} \mathrm{O}$-Narkoseschwelle durchgefïhrt. Stoffwechselmessungen mit dem Grundumsatzgerät der Firma Hartmann und Braun A.G., Frankfurt a. M., liefen den Narkoseversuchen parallel. Die $\mathrm{N}_{2} \mathrm{O}$-Narkoseschwelle und der Stoffwechsel unter Einwirkung von 2,4-Dinitrophenol (DNP) wurden $25 \mathrm{~min}$ nach der Injektion gemessen.

Die Resultate sind in Tabelle 1 zusammengefaßt. Zwischen der 4, und 5. Woche nach Entnahme der Schilddrüse liegt in allen Fällen die Narkoseschwelle eindeutig unter dem Ausgangswert. Der $\mathrm{O}_{2}$-Verbrauch ist von $330 \mathrm{ml} \mathrm{O} / \mathrm{kg} / \mathrm{h}$ auf $198 \mathrm{ml} \mathrm{O}_{2} / \mathrm{kg} / \mathrm{h}$, d.h. um $40 \%$ abgefallen. Die Narkoseschwelle läßt sich mit Thyroxin $(0.5 \mathrm{mg} / \mathrm{kg}$ s.c. täglich 8 Tage lang) 